\title{
A novel polystyrene-supported titanium dioxide photocatalyst for degradation of methyl orange and methylene blue dyes under UV irradiation
}

\author{
Seema Singh ${ }^{1 *}$, Aniket Chaki ${ }^{1}$, Devesh Pratap Chand ${ }^{1}$, Avinash Raghuwanshi ${ }^{1}$, Pramod Kumar Singh ${ }^{1}$ and Hari \\ Mahalingam $^{1 *}$ \\ ${ }^{1}$ Department of Chemical Engineering, Jaypee University of Engineering and Technology, Guna, Madhya Pradesh, \\ India.
}

\begin{abstract}
The commercialization of titanium dioxide-based heterogeneous photocatalysis continues to suffer from various limitations, the major shortcoming being the costly and time consuming post-treatment separation of very fine titanium dioxide particles. In order to eliminate this major hindrance, immobilization of titanium dioxide particles on various substrates continues to be an active area of research. In this work, polystyrene-supported titanium dioxide photocatalyst was prepared using a facile method. The photocatalytic activity of the developed photocatalysts was investigated by photodegradation of aqueous solutions of methylene blue andmethyl orange dyes under UV light for 24 $\mathrm{h}$ under non-stirred conditions. The recovery and reuse of the prepared photocatalysts was also investigated. The maximum percentage degradation of methyl orange and methylene blue dyes by the developed photocatalysts was found to be around $60 \%$ and $66 \%$ respectively. The ease of separation after use in addition to a facile, low cost-based method of fabrication and appreciable photocatalytic activity of the developed photocatalyst makes it a promising candidate to be explored further for large scale applications.
\end{abstract}

\section{INTRODUCTION}

In 1972, Akira Fujishima and Kenichi Honda first reported the photoinduced decomposition of water on titanium dioxide $\left(\mathrm{TiO}_{2}\right)$ electrodes. Since then, heterogeneous photocatalysis using semiconductor titanium dioxide photocatalyst has been an active and ever increasing area of research $[1,2]$.

$\mathrm{TiO}_{2}$ has been reported as one of the most efficient and promising photocatalyst because of the following properties: (i) high physical and chemical stability, (ii) strong oxidizing power, (iii) high photocatalytic activity, (iv) non-toxic nature, (v) high turnover, (vi) complete mineralization of organic pollutants, (vii) economical, (viii) relatively low band gap, (ix) can be supported on various substrates and (x) stability against photo corrosion [3-7].

Although the $\mathrm{TiO}_{2}$ photocatalyst is considered to be an ideal photocatalyst yet it suffers from two major limitations:

1) It is active only under UV light.

2) Recombination of photo-generated holes $\left(\mathrm{h}^{+}\right)$and electrons $\left(\mathrm{e}^{-}\right)$that leads to reduction in the overall quantum efficiency $[8,9]$.

For large scale commercial applications of $\mathrm{TiO}_{2}$ photocatalysis, extensive research is being carried out to modify $\mathrm{TiO}_{2}$ so as to make it effective in solar light that is freely and abundantly available. Some of the various approaches being tried for making titanium dioxide effective in visible light are doping, capping, dye sensitization, coupling and surface modification by noble metals $[3,5]$.
$\mathrm{TiO}_{2}$ is conventionally available in the form of powder. It can be applied to wastewater either in the form of powder, i.e., in slurry form or can be supported over a suitable substrate. Although when used in the form of powder, it shows greater surface area and efficiency yet it suffers from a major obstacle of post-treatment recovery that is both time and money consuming. Moreover, the light utilization efficiency of suspended photocatalyst is lowered due to the attenuation loss suffered by light raysas it travels in the water surface. To overcome these drawbacks, continuous efforts are being made to immobilize $\mathrm{TiO}_{2}$ on various substrates [5, 10].

In the literature, various substrates for supporting titanium dioxide photocatalyst have been reported. Few of these are inorganic carbon-fabrics [11], Indium tin oxide glass [12], plastics [13],natural fabrics [14], synthetic fabrics [15],polymers [16], glass mats [17], glass plates, raschig rings [18], fly ash, vycor glass, hollow glass spheres, reactor walls, fiber glass, silica gel, fabric or wool, anodised iron, micro-porous cellulose membranes, quartz optical fibres, zeolites, alumina clays, ceramic membranes and monoliths, stainless steel [19].

Of the above stated substrates,the polymer substrates for supporting titanium dioxide photocatalyst have been attracting a lot of recent attention. This is attributed to their following unique properties: (i) chemical inertness, (ii) mechanical stability, (iii) high durability, (iv) hydrophobicnature, (v) low cost, (vi) ease of availability, (vii) thermosoftening properties, (viii) high UV-resistance and (ix) low density for most of them [3]. 
A variety of methods for supporting titanium dioxide on polymer substrate have been reported in the literature. Some of them are sol-gel method [20], chemical vapour deposition (CVD) [21] thermal treatment method [22], hydrothermal methods [23], sol-spray methods [24] and electrophoretic deposition [25].

Due to the need of high temperature calcination along with complex procedures and expensive instruments, most of the above mentionedtechniques do not find their use in coating polymer substrate with $\mathrm{TiO}_{2}$. As per our literature survey, it appears that sol-gel and sputtering have been the main low-temperature deposition techniques for supporting titanium dioxide nanoparticleson various polymeric substrates. Although sol-gel methodis very convenient yet the photocatalytic activities of $\mathrm{TiO}_{2}$ films supported on substrates by this method are limited. This is due to the fact that efficient photo-induced holes and electrons are generated only in well crystallized phases of titanium dioxide. To obtaindesired crystal phase from amorphous sol-gel $\mathrm{TiO}_{2}$ films, thermal treatment at relatively high temperatures(generally $300^{\circ} \mathrm{C}$ and above) is required post deposition. This restricts the applicationof sol-gel method for supporting titania only on those polymeric substrates that have good thermal stability [3].

In this paper, a novel, facile and low cost-based method is used to immobilize commercial $\mathrm{TiO}_{2}$ powder on polystyrene (PS) substrate. For photocatalytic degradation studies, methylene blue (MB) and methyl orange (MO) dyes were employed as model organic dye compounds. The photodegradation of MB and MO dyes by the prepared polystyrene-supported titanium dioxidephotocatalysts was investigated.

\section{EXPERIMENTAL DETAILS}

\subsection{Materials, Equipment and Methods}

The chemicals used in this study were: xylene (AR,SDFCL), polystyrene beads(Sigma-Aldrich), P25 $\mathrm{TiO}_{2}$ (Evonik Degussa AG, Germany; BET surface area $\left.50 \pm 15 \mathrm{~m}^{2} \mathrm{~g}^{-1}\right)$, methylene blue $\left(\mathrm{C}_{16} \mathrm{H}_{18} \mathrm{~N}_{3} \mathrm{SCl}\right.$, Merck, India), methyl orange $\left(\mathrm{C}_{14} \mathrm{H}_{14} \mathrm{~N}_{3} \mathrm{NaO}_{3} \mathrm{~S}\right.$, Merck, India). Distilled water was used throughout this study. Images were obtained by using an optical microscope (NIKON Eclipse E600 POL). The $\mathrm{TiO}_{2}$ slurry samples were centrifuged using REMI R-8C Laboratory centrifuge. The calibration curves for MB and MO dyes and their photodegradation under UV illumination (Hg lamp, 254 nm, 15W, Philips) was investigated with the help of UV-visspectrophotometer (ELICO SL159).

\subsection{Preparation of $\left(\mathrm{TiO}_{2} / \mathrm{PS}\right)$ photocatalysts}

The $\mathrm{TiO}_{2} / \mathrm{PS}$ photocatalysts were prepared by mixing $\mathrm{TiO}_{2}$ (Degussa P25) powder in polystyrene (PS) dissolved in xylene at $80^{\circ} \mathrm{C}$. Different $\mathrm{TiO}_{2}$ samples, namely $\mathrm{TiO}_{2(0.02)} / \mathrm{PS}, \mathrm{TiO}_{2(0.03)} / \mathrm{PS}, \mathrm{TiO}_{2(0.04)} / \mathrm{PS}$ were prepared by taking $0.02 \mathrm{~g}, 0.03 \mathrm{~g}$ and $0.04 \mathrm{~g}$ amount of $\mathrm{TiO}_{2}$ respectively.The suspension was casted into a petridish and left at room temperature for solvent to evaporate. Then the petridish was kept in an oven at $80^{\circ} \mathrm{C}$ for about $20 \mathrm{~h}$ to obtain a thick sheet of photocatalyst. The sheets were washed several times with distilled water, air-dried and stored.

\subsection{Preparation of calibration curves for $\mathrm{MB}$ and MO}

Different concentrations of MB and MO solutions were prepared from stock solutions of $50 \mathrm{ppm}$ and the calibration curves for $\mathrm{MB}$ and $\mathrm{MO}$ solutions were obtained by using UV-vis spectrophotometer at absorption maximum of $664 \mathrm{~nm}$ and $465 \mathrm{~nm}$ respectively.

\subsection{Photocatalytic study}

During the photocatalytic study, small pieces of $\mathrm{TiO}_{2} / \mathrm{PS}$ photocatalyst samples weighing $3 \mathrm{~g}$ were placed in a $100 \mathrm{~mL}$ beaker containing $50 \mathrm{~mL}$ of $\mathrm{MB}$ solution $(5 \mathrm{ppm})$. A similar setup was used for studying the photodegradation of MO dye. The solutions were then kept in dark for a period of $60 \mathrm{~min}$ to reach adsorption equilibrium. To avoid oxygenation of the solutions, no stirring was used in the reactions. After $1 \mathrm{~h}$ of adsorption period, the initial absorbance was recorded at a wavelength of $664 \mathrm{~nm}$ for MBsolution and $465 \mathrm{~nm}$ for MO solution. Subsequently, the irradiation was started by turning on the UV light kept at $10 \mathrm{~cm}$ above the solution surface. The photodegradation of $\mathrm{MB}$ and MO solutions was analysed after $6 \mathrm{~h}, 18 \mathrm{~h}$ and $24 \mathrm{~h}$ of UV irradiation. For this, $5 \mathrm{~mL}$ sample of $\mathrm{MB}$ and MO solutions was retrieved from the beaker, analyzed and then returned to the beaker.

A comparative study employing a similar setup was also undertaken using $0.02 \mathrm{~g}$ of commercial $\mathrm{TiO}_{2}$ (Degussa $\mathrm{P} 25)$ in slurry form. The solution samples retrieved from the beaker were centrifuged to obtain clear solutions before spectrophotometric analysis.

\section{ObServations, Results AND DISCUSSIONS}

\section{1 $\mathrm{TiO}_{2} / \mathrm{PS}$ photocatalyst characterization}

The preliminary images (Fig. 1 ) of the prepared $\mathrm{TiO}_{2} / \mathrm{PS}$ photocatalysts were obtained by using an optical microscope. The images suggest the presence of $\mathrm{TiO}_{2}$ particles on the polystyrene surface. The $\mathrm{TiO}_{2} / \mathrm{PS}$ photocatalyst sample with higher loading amount of $\mathrm{TiO}_{2}$ show higher $\mathrm{TiO}_{2}$ agglomerates. Further investigation using electron microscopy (SEM/TEM) needs to be undertaken. 



Fig.1: Optical microscope images of $\mathrm{TiO}_{2} / \mathrm{PS}$ photocatalysts on $20 \mathrm{X}$ magnification scale

(a) $\mathrm{TiO}_{2(0.02)} / \mathrm{PS}$, (b) $\mathrm{TiO}_{2(0.03)} / \mathrm{PS}$, (c) $\mathrm{TiO}_{2(0.04)} / \mathrm{PS}$

\subsection{Calibration curves for MB and MO}

Straight lines were obtained for the calibration curve of both $\mathrm{MB}$ and $\mathrm{MO}$ solutions (with $\mathrm{R}^{2}=0.9937$ and 0.9997 respectively)(Fig. 2).
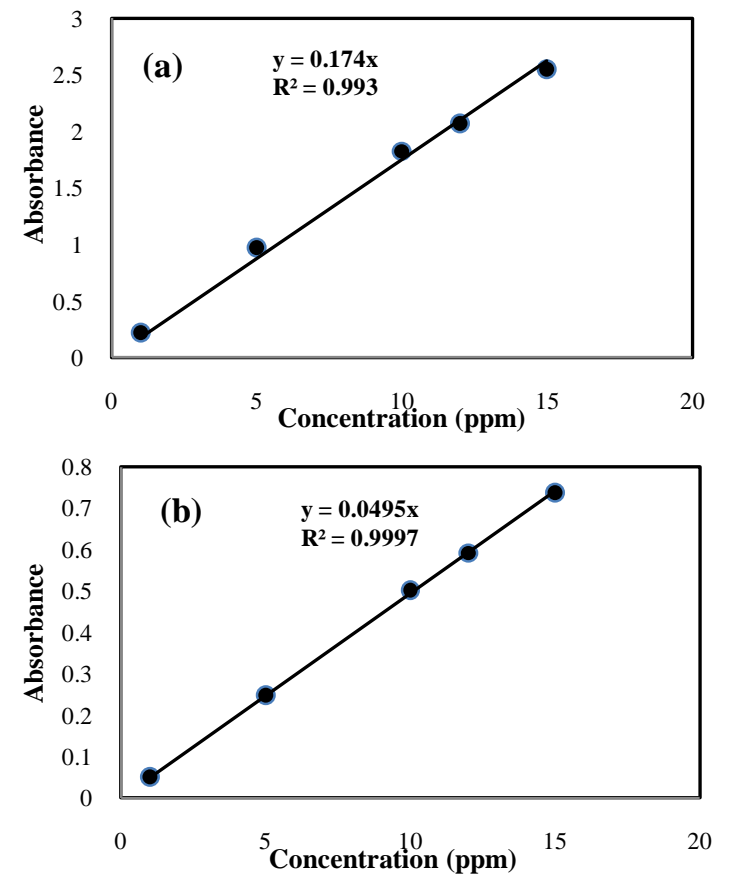

Fig. 2: Calibration curves for (a) methylene blue, (b) methyl orange

\subsection{Photocatalytic reactions}

After $1 \mathrm{~h}$ of adsorption period in the dark, it was found out that the $\mathrm{TiO}_{2(0.02)} / \mathrm{PS}$ sample showed lowerabsorbance as compared to $\mathrm{TiO}_{2(0.03)} / \mathrm{PS}$, $\mathrm{TiO}_{2(0.04)} / \mathrm{PS}$ and $\mathrm{TiO}_{2}$ (Degussa P25, slurry form) photocatalyst samples. This could be attributed to the lower adsorption of the dye molecules by relatively lesser amount of $\mathrm{TiO}_{2}$ particles present in $\mathrm{TiO}_{2(0.02)} / \mathrm{PS}$ sample. In the absence of the photocatalyst in $\mathrm{MB}$ and MO solutions (blank experiment-I) as well as in presence of only PS beads in MB and MO solutions (blank experiment-II), no significant discoloration of MB and MO solutions under UV irradiation was observed. This confirmed that the photocatalytic activity was due to $\mathrm{TiO}_{2}$ particles present in the PS substrate.

The photocatalytic studies were carried out by investigating the degradation of dyes, MB and MO as organic probe molecules under UV light irradiation after $6 \mathrm{~h}, 18 \mathrm{~h}$ and $24 \mathrm{~h}$. It was observed that the prepared $\mathrm{TiO}_{2} / \mathrm{PS}$ photocatalysts could degrade both the MB and MO. The percentage degradation for all the three prepared photocatalysts increased as the irradiation time increased from $6 \mathrm{~h}$ to $18 \mathrm{~h}$ and subsequently $24 \mathrm{~h}$. The experimental data for the percentage degradation of MBand MO solutions by different samples of the photocatalystsis shown in Fig. 3.

The percentage degradation of MO and MB solutions by various prepared samples of $\mathrm{TiO}_{2} / \mathrm{PS}$ photocatalysts under UV irradiation $(\lambda=254 \mathrm{~nm})$ was found to be around $60 \%$ and $66 \%$ respectively. The percentage degradation of MO solution increased with time as well as loading amount of $\mathrm{TiO}_{2}$ in the photocatalyst. For MB solution, $\mathrm{TiO}_{2(0.02)} / \mathrm{PS}$ and $\mathrm{TiO}_{2(0.03)} / \mathrm{PS}$ photocatalyst samples showed almost similar degradation efficiencies but $\mathrm{TiO}_{2(0.04)} / \mathrm{PS}$ photocatalyst exhibitedslightly lesser percentage degradation for MB. The result suggests that the amount of $\mathrm{TiO}_{2}$ has a significant influence on the photocatalytic activity of the photocatalyst and thus, optimization of loading amount of $\mathrm{TiO}_{2}$ in the photocatalyst needs further investigation. For comparison, a similar experimental setup was made to investigate the photocatalytic degradation by $0.02 \mathrm{~g}$ of $\mathrm{TiO}_{2}$ (Degussa P25)in slurry form. It was observed that the slurry form of titaniacompletely degraded both, the $\mathrm{MB}$ and MO solutions, within $2.5 \mathrm{~h}$ of UV irradiation. This could be attributed to the greater surface area offered by the photocatalysts in powder form as compared with the supported forms.

On investigation, it was found the polystyrenesupported $\mathrm{TiO}_{2}$ photocatalysts scored over slurry form of $\mathrm{TiO}_{2}$ in ease of post-treatment recovery. Recyclability of the prepared $\mathrm{TiO}_{2} / \mathrm{PS}$ photocatalysts was also investigated by using the $\mathrm{TiO}_{2} / \mathrm{PS}$ photocatalysts (without any intermittent cleaning) for degradation of fresh MO dye solutions. It was observed that after second reaction run, the photocatalytic activity of the $\mathrm{TiO}_{2} / \mathrm{PS}$ sheets decreased only slightly. 

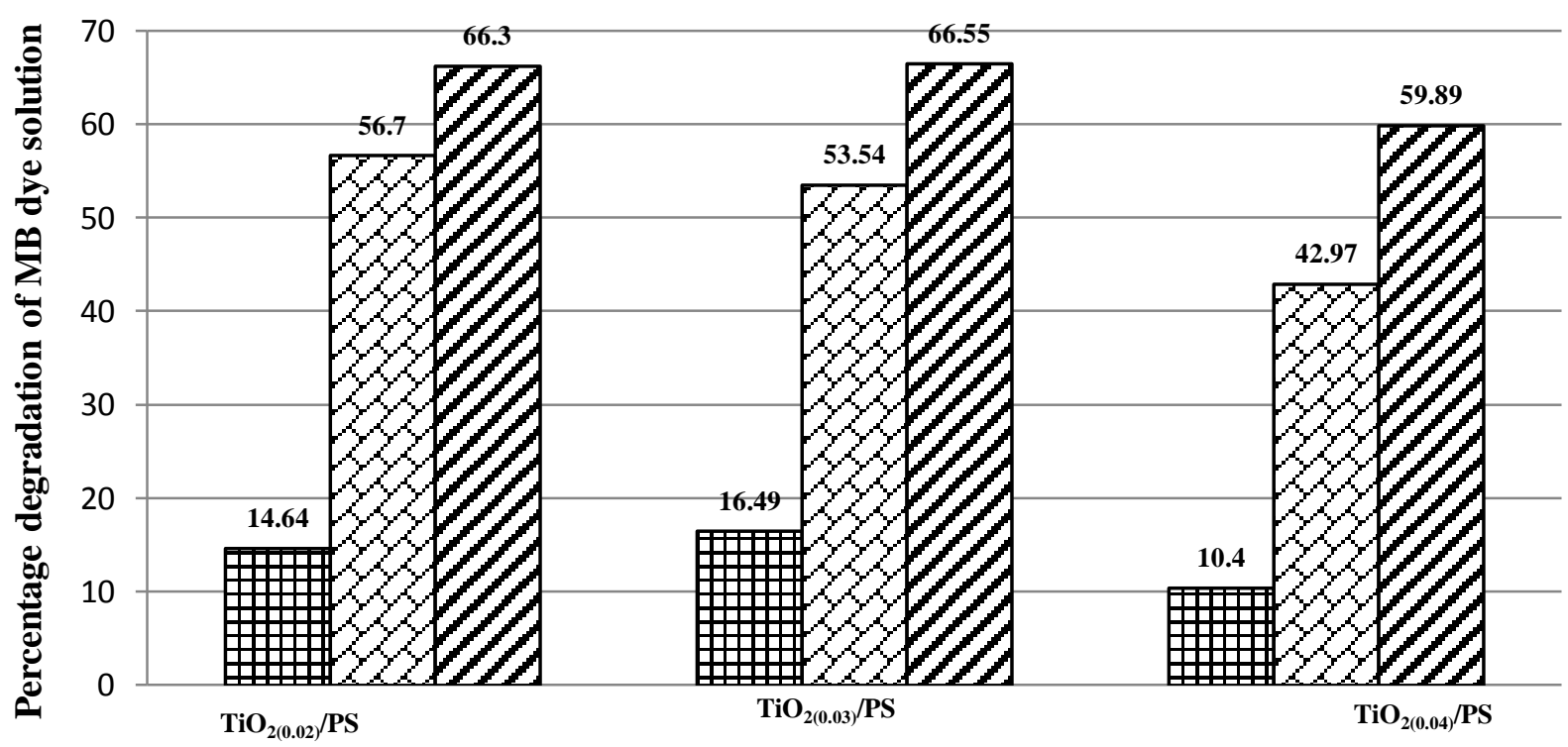

Sample details

$\boxplus$ After 6h of UV irradiation $\square$ After 18h of UV irradiation $\square$ After 24h of UV irradiation

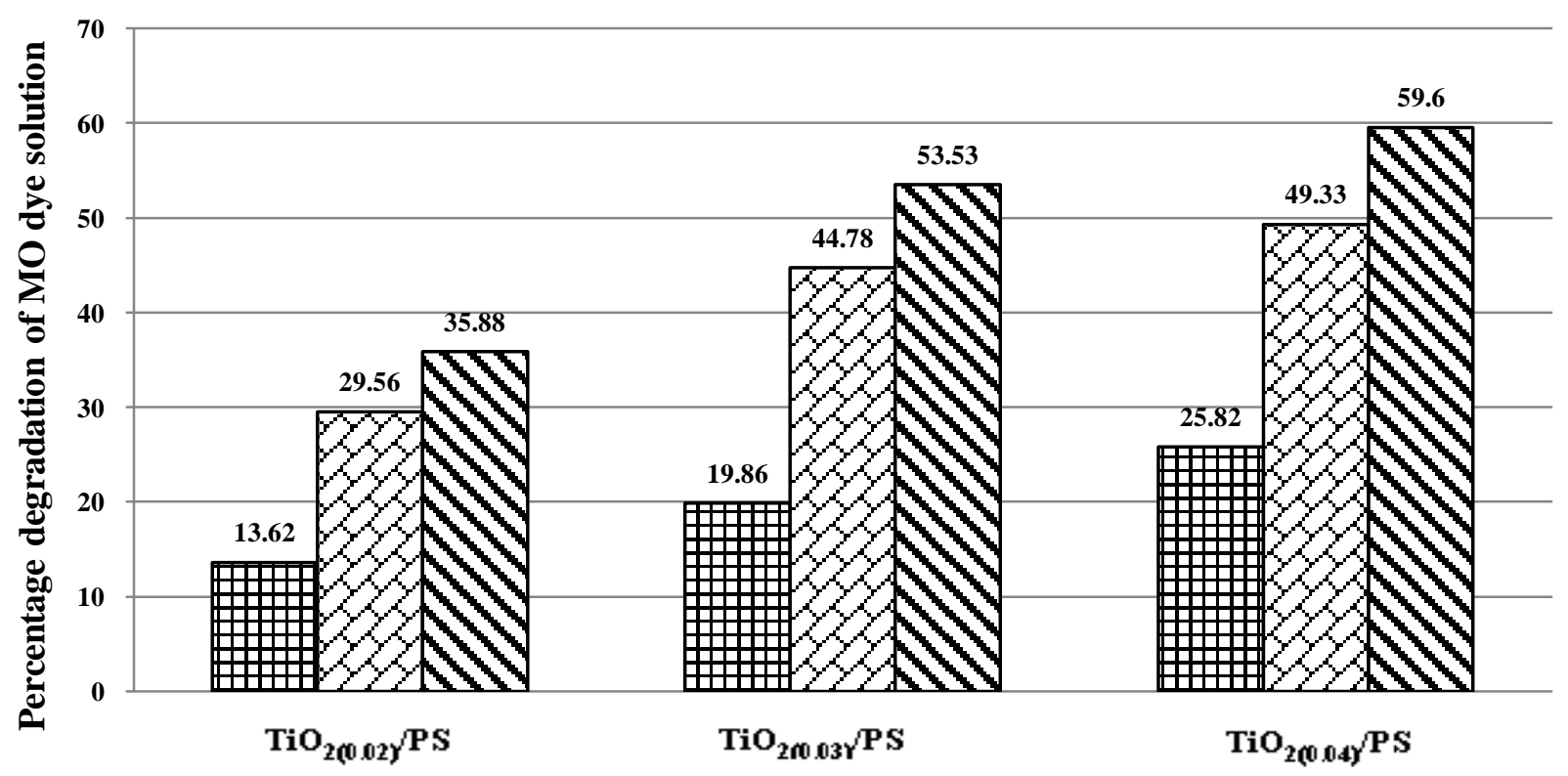

Sample details

日After 6h of UV irradiation $\square$ After 18h of UV irradiation $\square$ After 24h of UV irradiation

Fig. 3: Photodegradation of $\mathrm{MB}$ and $\mathrm{MO}$ dye solutions by prepared $\mathrm{TiO}_{2} / \mathrm{PS}$ photocatalysts.

\section{CONCLUSIONS}

A novel and simple method for preparing polystyrenesupported $\mathrm{TiO}_{2}$ photocatalysts has been described. All the prepared $\mathrm{TiO}_{2} / \mathrm{PS}$ photocatalyst samples could degrade both the MBand MOsolutions under UV irradiation. The percentage degradation by the $\mathrm{TiO}_{2} / \mathrm{PS}$ photocatalysts was lower than that exhibited by slurry form of $\mathrm{TiO}_{2}$. However, the ease of separation after use in addition to a facile and low cost-based method of fabrication and appreciable photocatalytic activity of the developed polystyrene-supported $\mathrm{TiO}_{2}$ photocatalyst makes it an attractive and promising candidate to be 
explored further for large scale applications like degradation of recalcitrant pollutants from wastewater. Due to the promising nature of the prepared polystyrene-supported $\mathrm{TiO}_{2}$ photocatalysts, further investigation in terms of characterizing the physicochemical properties of the photocatalysts using scanning electron microscopy, optimization and influence of various parameters like amount of titanium dioxide in the photocatalyst, $\mathrm{pH}$ and temperature of the dye solution and recyclability over multi-reaction runs needs to be undertaken.

\section{REFERENCES}

[1] A. Fujishima, K. Honda, Nature 238 (1972) 37-38.

[2] K. Hashimoto, H. Irie, A. Fujishima, AAPPS Bulletin 17 (6)(2007) 12-28.

[3] S. Singh, H. Mahalingam, P.K. Singh, Appl. Catal., A 462- 463 (2013) 178- 195.

[4] S.M. Gupta, M.Tripathi, Chinese Sci Bull, 56 (16) (2011) 1639-1657.

[5] H.Han, R.Bai, Ind. Eng. Chem. Res. 48 (2009) 2891-2898.

[6] J. Kasanen, J. Salstela, M. Suvanto, T.T.Pakkanen, Appl. Surf. Sci. 258 (2011) 1738- 1743.

[7] J. Kasanen, M. Suvanto, T.T. Pakkanen, J.Appl.Polym. Sci. 119 (2011) 2235-2245.

[8] M. Pelaez, N.T. Nolan, S.C. Pillai , M. K. Seery, P. Falaras, A.G. Kontos, P.S.M. Dunlop, J.W.J. Hamilton, J.A. Byrne, K.O'Shea, M.H. Entezari, D.D. Dionysiou, Appl. Catal., B 125 (2012) 331349.

[9] U.G. Akpan, B.H. Hameed, J. Hazard. Mater. 170 (2009) 520-529.

[10] J.A. Byrne, B.R. Eggins, N.M.D. Brown, B. McKinney, M. Rouse, Appl.Catal.,B 17 (1998) 2536.
[11] T. Yuranova, O. Enea, E. Mielczarski, J. Mielczarski, P. Albers, J. Kiwi, Appl. Catal., B 49 (2004) 39-50.

[12] S.B. Sankapal, M.Ch. Steiner, A. Ennaoui, Appl. Surf. Sci. 239 (2005) 165-170.

[13] C.H. Kwon, H. Shin, J.H. Kim, W.S. Choi, K.H. Yoon, Mater. Chem. Phys. 86 (2004) 78-82.

[14] T. Yuranova, R. Mosteo, J. Bandara, D. Laub, J. Kiwi, J. Mol. Catal. A 244 (2006) 160-167.

[15] A. Bozzi, T. Yuranova, J. Kiwi, J. Photochem. Photobiol. A 172 (2005) 27-34.

[16] J.H. Yang, Y.S. Han, J.H. Choy, Thin Solid Films 495 (2006) 266-271.

[17] M. Dhananjeyan, J. Kiwi, P. Albers, O. Enea, Helv. Chim. Acta 84 (2001) 3433-3445.

[18] Y. Zhiyong, H. Keppner, D. Laub, E. Mielczarski, J. Mielczarski, L.K-Minsker, A. Renken, J. Kiwi, Appl.Catal., B 79 (2008) 63-71.

[19] M.E. Fabiyi, R.L. Skelton, J. Photochem. Photobiol., A 132 (2000) 121-128.

[20] Y. Chen, E. Stathatos, D.D. Dionysiou, Surf. Coat. Technol. 202 (2008) 1944-1950.

[21] C. Kuo, Y. Tseng, C. Huang, Y. Li, J. Mol. Catal. A 270 (1-2) (2007) 93-100.

[22] K. Tennakone, C.T.K. Tilakaratne, I.R.M. Kottegoda, J. Photochem. Photobiol., A 87 (1995) 177-179.

[23] S. Liu, X. Chen, J. Chem. Technol. Biotechnol. 82 (2007) 453-459.

[24] A.Y. Shan, T.I.M. Ghazi, S.A. Rashid, Appl. Catal., A 389 (2010) 1-8.

[25] M.S. Djosic, V.B. Miskovic-Stankovic, Dj. T. Janackovic, Z.M. Kacarevic-Popovic, R.D. Petrovic, Colloids Surf. A 274 (2006) 185-191. 